\title{
Hybrid band offset calculation for heterojunction interfaces between disparate semiconductors
}

Cite as: Appl. Phys. Lett. 116, 131602 (2020); https://doi.org/10.1063/1.5135376

Submitted: 06 November 2019 . Accepted: 13 March 2020 . Published Online: 30 March 2020

Zhaofu Zhang $(\mathbb{D})$, Yuzheng Guo $(\mathbb{D})$, Haichang Lu $(\mathbb{D})$, Stewart J. Clark $(\mathbb{D}$, and John Robertson
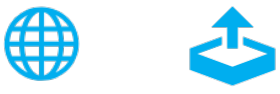

\section{ARTICLES YOU MAY BE INTERESTED IN}

Ultrafast dynamics of hole self-localization in $\beta-\mathrm{Ga}_{2} \mathrm{O}_{3}$

Applied Physics Letters 116, 132101 (2020); https://doi.org/10.1063/5.0003682

Spin-transport in superconductors

Applied Physics Letters 116, 130501 (2020); https://doi.org/10.1063/1.5138905

Oxygen vacancies: The (in)visible friend of oxide electronics

Applied Physics Letters 116, 120505 (2020); https://doi.org/10.1063/1.5143309

\section{Hall Effect Measurement Handbook}

A comprehensive resource for researchers Explore theory, methods, sources of errors, and ways to minimize the effects of errors

\section{Request it here}

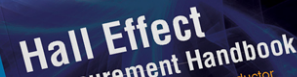

Measurement Handi

Lake Shore
CRYOTRONICs

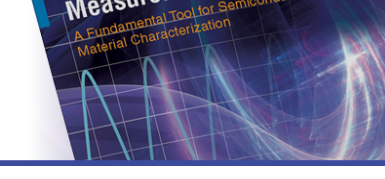




\title{
Hybrid band offset calculation for heterojunction interfaces between disparate semiconductors
}

\author{
Cite as: Appl. Phys. Lett. 116, 131602 (2020); doi: 10.1063/1.5135376 \\ Submitted: 6 November 2019 • Accepted: 13 March 2020 . \\ Published Online: 30 March 2020
}

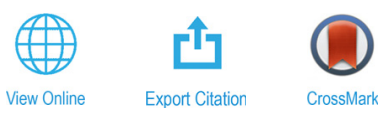

Zhaofu Zhang, ${ }^{1}$ (D) Yuzheng Guo, ${ }^{2}$ (D) Haichang Lu, (D) Stewart J. Clark, ${ }^{3}$ (D) and John Robertson ${ }^{1, a)}$

AFFILIATIONS

${ }^{7}$ Department of Engineering, University of Cambridge, Cambridge CB2 1PZ, United Kingdom

${ }^{2}$ College of Engineering, Swansea University, Swansea SA1 8EN, United Kingdom

${ }^{3}$ Department of Physics, University of Durham, Durham DH1 3LE, United Kingdom

a) Author to whom correspondence should be addressed: jr@eng.cam.ac.uk

\begin{abstract}
Accurate band offset calculations are challenging for heterojunction interfaces that consist of two very different host materials. For this, the key requirement is to have the correct bandgap of each material at the same time. A hybrid calculation scheme (HSE/-U scheme) is proposed to model the band offsets of such interfaces. Our HSE/-U method applies the hybrid functional for the whole interface supercell, but with an additional "reverse GGA $+U$ " on the narrow gap semiconductor side, guaranteeing the correct bandgaps on both sides. Several supercell calculations of dielectric films including $\mathrm{HfO}_{2}, \mathrm{ZrO}_{2}, \mathrm{Al}_{2} \mathrm{O}_{3}, \mathrm{TiO}_{2}$, and $\mathrm{GaN}$ on an insulating phase $\mathrm{VO}_{2}$ are tested to verify it. All the studied oxides show the type-I band alignment with $\mathrm{VO}_{2}$, and the band edge line-up agrees well with the available experimental reports, supporting the reliability of the proposed hybrid calculation scheme.
\end{abstract}

Published under license by AIP Publishing. https://doi.org/10.1063/1.5135376

The band offset (BO) of a semiconductor heterojunction is the key parameter that determines its electrical behavior. ${ }^{1,2}$ BOs can be calculated using density functional theory (DFT) and a supercell atomic model of the interface. However, such calculations using either the local density approximation (LDA) or the generalized gradient approximation (GGA) can lead to significant gap underestimation, impairing the reliability of the calculated BOs. Typically, to keep computational costs within limits, these errors are overcome by using hybrid functionals to represent the electronic exchange-correlation functional. These functionals mix a certain fraction (i.e., $\alpha$ ) of Hartree-Fock (HF) exchange. The chosen hybrid functional will use the same parameter set to describe both sides of the interface, particularly if a plane wave basis set is used. This works when the chosen hybrid functional is PBE0, $\mathrm{B} 3 \mathrm{LYP},{ }^{5}$ or screened-exchange (sX), ${ }^{6}$ where their bandgap value agrees with the experimental value over a range of gap magnitudes. However, for range-separated hybrids such as the Heyd-Scuseria-Ernzerhof (HSE) hybrid, ${ }^{7}$ it is found that an increasing HF fraction must be included for the calculated bandgap to agree with experiment for wider bandgaps. $^{8-10}$ Clearly, this could lead to difficulties when studying heterojunctions between semiconductors with widely different gaps, such as a semiconductor and a wide gap insulator.

The heterojunctions of $\mathrm{VO}_{2}$ have a related problem. $\mathrm{VO}_{2}$ is a correlated oxide with a first-order metal-insulator transition (MIT), which makes it of technological interest ${ }^{11}$ for smart windows, RF or optical switches, "Mott-FETs" (MIT FETs), ${ }^{12}$ and steep slope devices. ${ }^{13}$ These devices have an interface between $\mathrm{VO}_{2}$ and a semiconductor like $\mathrm{GaN},{ }^{14-16}$ a substrate like $\mathrm{TiO}_{2},{ }^{13}$ or a gate insulator like $\mathrm{HfO}_{2} \cdot{ }^{17,18}$ The electronic structure of $\mathrm{VO}_{2}$ can be described by methods such as GW or cluster-dynamic mean field theory (c-DMFT). ${ }^{19,20}$ On the other hand, hybrid functionals describe many aspects of its electronic structure within band theory at a relatively low cost. ${ }^{21}$ However, it was recently noted that hybrid functionals over-estimate the gap of the lowtemperature phase, also resulting in incorrect magnetic ordering. ${ }^{22-25}$ These problems could be minimized by using a smaller empirical fraction of Hartree-Fock exchange, $\alpha \sim 0.10$ or less, rather than the standard value of $\alpha=0.25 .^{22-27}$ Thus, a typical $\mathrm{VO}_{2}$ interface calculation would need to use different values of $\alpha$ on each side of the interface.

Before the advent of hybrid functionals, an improvised method to correct the DFT bandgap error in some correlated electron systems was to include an on-site repulsion on $d$ orbitals. ${ }^{28,29}$ This GGA $+\mathrm{U}$ method originated for open-shell systems, but it was also used empirically for closed-shell systems like $\mathrm{ZnO}$ and $\mathrm{GaN}$, ${ }^{29}$ where $\mathrm{U}$ would push down the filled cation $d$ states, thereby lowering the anion $p$-like valence band maximum (VBM) and widening the bandgap.

Here, we show that a combination of a hybrid functional and a $\mathrm{GGA}+\mathrm{U}$ method with a negative $\mathrm{U}$ term can be used to independently 
tune the bandgap on both sides of an interface and thus model heterojunctions of more disparate systems. For the dielectric/ $\mathrm{VO}_{2}$ case, we use the HSE functional with a certain mixing fraction to obtain the correct gap at the $\mathrm{HfO}_{2}$ insulator side, but this leads to a severely overestimated $\mathrm{VO}_{2}$ bandgap. Meanwhile, a reverse GGA+U with a negative $\mathrm{U}$ term on the $\mathrm{V}-d$ orbital is used to narrow the $\mathrm{VO}_{2}$ gap to its experimental value. With this hybrid calculation scheme, several types of dielectrics facing an insulating $\mathrm{M} 1$ phase $\mathrm{VO}_{2}$ can be calculated. The band alignments obtained by this method agree well with experimental values, indicating a reliable calculation scheme. They also allow the correct latent heat of the rutile and $\mathrm{M} 1 \mathrm{VO}_{2}$ phases to be described, ${ }^{24}$ which a pure HSE calculation would not. A correct value of the R-M1 phase free energy difference or "latent heat" is critical for a correct microscopic description of the Mott-FET. ${ }^{25}$

The calculations use the plane wave code $\operatorname{CASTEP}^{30}$ with normconserving pseudopotentials and a plane wave cutoff energy of $680 \mathrm{eV}$, which converged total energy differences to $1 \mathrm{meV}$ /atom. A $5 \times 5 \times 1$ $k$-point mesh was adopted in the calculation. The GGA-PBE exchange-correlation functional was employed to relax the geometry. The electronic calculation for interface supercells was performed with the HSE/-U scheme. It is noted that the hybrid functional and $\mathrm{GGA} \pm \mathrm{U}$ calculations were conducted simultaneously. For comparison, HSE calculations with a $10 \%$ HF fraction were also conducted. The ground state antiferromagnetic ordering for the $\mathrm{VO}_{2} \mathrm{M} 1$ phase is used, as previously. ${ }^{31}$

Since the essential requirement for interface modeling is the local bonding rather than long-range crystalline symmetry, the monoclinic $\mathrm{M} 1$ phase $\mathrm{VO}_{2}$ was strained to be orthorhombic to match the crystalline insulators. Several dielectrics are considered, namely, high dielectric constant oxide $\mathrm{HfO}_{2}$ (and $\mathrm{ZrO}_{2}$ ), wide bandgap oxide $\mathrm{Al}_{2} \mathrm{O}_{3}$, narrower gap oxide $\mathrm{TiO}_{2}$, and wide gap semiconductor $\mathrm{GaN}$. $\theta-\mathrm{Al}_{2} \mathrm{O}_{3}$ was used rather than the hexagonal $\alpha-\mathrm{Al}_{2} \mathrm{O}_{3}$ phase (sapphire), ${ }^{32}$ due to its comparable properties to amorphous $\mathrm{Al}_{2} \mathrm{O}_{3} \cdot{ }^{32,33}$ For interface modeling, $\theta-\mathrm{Al}_{2} \mathrm{O}_{3}$ was strained into an orthorhombic structure to match the $\mathrm{M} 1$ phase of $\mathrm{VO}_{2}$. Cubic $\mathrm{HfO}_{2}$ (and $\mathrm{ZrO}_{2}$ ) and anatase $\mathrm{TiO}_{2}$ can contact $\mathrm{VO}_{2}$ with a negligible mismatch. Considering that rutile $\mathrm{TiO}_{2}\left(\mathrm{r}-\mathrm{TiO}_{2}\right)$ is often used in experiments as a substrate for $\mathrm{VO}_{2}$ when using straining engineering, ${ }^{13}$ the $\mathrm{r}-\mathrm{TiO}_{2} / \mathrm{M} 1-\mathrm{VO}_{2}$ interface was also studied, despite the larger lattice mismatch between the two hosts (16\% and $10 \%$ mismatch in $x$ - and $y$-directions, respectively).

To build the interface model, a five-bilayer (001) surface slab of oxide is stacked on top of a five-bilayer $\mathrm{VO}_{2}(001)$ slab. The slab models are thick enough after the convergence test to well present the bulk atom properties. The interface models are built based on the principle of maximizing the fraction of heteropolar bonds, and that bonding at interfacial oxygen sites should resemble the bulk coordination (Fig. 1). The metal-oxygen interfacial bonding is also tested to be energetically favorable. The electron counting rule is applied to ensure an insulating interface. $^{33,34}$ We use a two-interface model without a vacuum gap and passivation layer for the oxide systems, and the supercells are allowed to relax in the $z$-direction to release internal stress. For the $\mathrm{GaN} / \mathrm{VO}_{2}$ interface, the modeling strategy of Ref. 14 is adopted.

Figure 1 shows the relaxed dielectric/ $\mathrm{VO}_{2}$ interface structures, where only metal-oxygen bonds exist at the interface, without any metal-metal bonding. For $\mathrm{HfO}_{2} / \mathrm{VO}_{2}$, the interfacial $\mathrm{O}$ atoms are threefold coordinated, the same as its bonding in bulk $\mathrm{VO}_{2}$. The geometry of the $\mathrm{ZrO}_{2}$ interface is quite similar to the $\mathrm{HfO}_{2}$ case

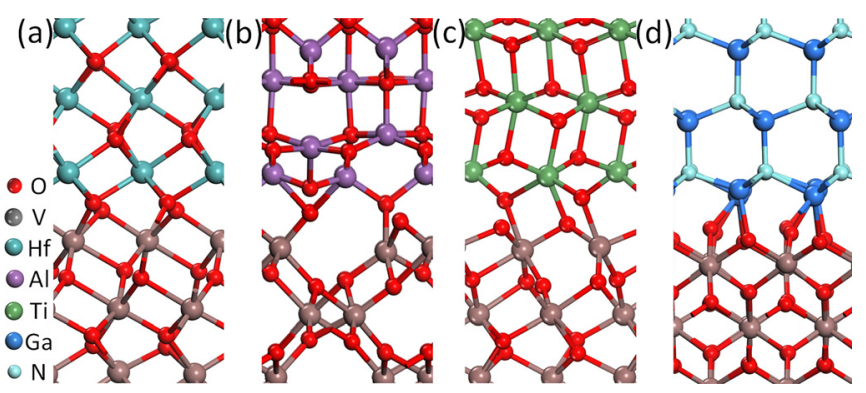

FIG. 1. Relaxed interface configurations of (a) $\mathrm{HfO}_{2} \mathrm{NO}_{2}$, (b) $\mathrm{Al}_{2} \mathrm{O}_{3} \mathrm{NO}_{2}$, (c) a- $\mathrm{TiO}_{2} /$ $\mathrm{VO}_{2}$, and (d) $\mathrm{GaN} / \mathrm{VO}_{2}$.

(not shown), owing to their similar physical properties. For the $\mathrm{Al}_{2} \mathrm{O}_{3}$ interface, the interfacial $\mathrm{Al}$ atoms are either fivefold or fourfold and $\mathrm{O}$ atoms are threefold or twofold, under-coordinated compared to their bulk sites. The dangling bonds on interfacial $\mathrm{Al}$ and $\mathrm{O}$ atoms are compensated with each other to achieve a closed-shell structure with no gap states. This also perfectly satisfies the electron counting rule, ${ }^{33,34}$ and thus, an insulating interface is found. Similar undercoordination in the interfacial region is also found for $\mathrm{TiO}_{2} / \mathrm{VO}_{2}$ and $\mathrm{GaN} / \mathrm{VO}_{2}$. The relaxed interface structure of $\mathrm{r}-\mathrm{TiO}_{2} / \mathrm{VO}_{2}$ and its partial density of states (PDOS) are also calculated, just for comparing with the $\mathrm{a}-\mathrm{TiO}_{2}$ interface alignment.

The traditional HSE electronic calculations with different HF fractions ( $\alpha$ values) were performed first on bulk $\mathrm{VO}_{2}$ and bulk dielectrics. We found that for $\alpha=30 \%$, the wider gap oxide $\mathrm{HfO}_{2}, \mathrm{ZrO}_{2}$, and $\mathrm{Al}_{2} \mathrm{O}_{3}$ have correct bandgaps of $5.93 \mathrm{eV}, 5.53 \mathrm{eV}$, and $6.05 \mathrm{eV}$, respectively. With a $25 \%$ mixing, the narrower gap insulator a- $\mathrm{TiO}_{2}$ shows a bandgap of $2.97 \mathrm{eV}$. For GaN, an $\alpha$ value of $27 \%$ is used to obtain a gap of $3.4 \mathrm{eV} .{ }^{33}$ These HSE gaps agree well with previous hybrid calculations and experimental values. ${ }^{35}$ Although some differences to the experimental gaps still exist, the key focus is the validity of this proposed hybrid calculation scheme. Thus, these mentioned HF fractions are chosen for the corresponding HSE/-U interface calculation.

The HSE functional with large $\alpha$ leads to an overestimated $\mathrm{VO}_{2}$ gap, while the negative $U$ potential helps narrow the gap back to the experiment one. To understand the variation trends, we studied bulk $\mathrm{VO}_{2}$ with a series of $\alpha$ and $\mathrm{U}$ parameters at first, and its calculated band energies are summarized in Table I.

The partial density of states (PDOS) of $\mathrm{VO}_{2}$ bulk with 0.30/ $-4.2 \mathrm{eV} \mathrm{HSE/-U}$ parameters is shown in Fig. 2. The separation between the highest occupied V- $d_{\|}$state and O-p state is consistent with the traditional HSE conclusion $(\alpha \sim 0.1){ }^{21,26}$ The bonding $d_{\|}$ band has a bandwidth of $1 \mathrm{eV}$ centered at $\sim 0.6 \mathrm{eV}$ below the Fermi level, in good agreement with the photoemission value. ${ }^{36,37}$ With fixed $\alpha$, a more negative $\mathrm{U}$ value on the $\mathrm{V}-d$ orbital increases the $\mathrm{V}-d \mathrm{O}-\mathrm{p}$ separation gap and thus reduces the $\mathrm{VO}_{2}$ bandgap. We see that the $\mathrm{V}-d_{\|}$O-p separation gap $(\sim 1.4 \mathrm{eV})$ is still close to the experimental value $(\sim 1.0 \mathrm{eV})^{36,37}$ compared to pure GGA $+\mathrm{U}$ data. Thus, this $\mathrm{HSE} /-\mathrm{U}$ hybrid scheme correctly represents the $\mathrm{VO}_{2}$ gap and simultaneously maintains an acceptable band structure. Interestingly, the bonding $\mathrm{V}-d_{\|}$bandwidth is almost the same under different HSE/-U parameters because the Coulomb interaction only affects the relative orbital energy, not the orbital bandwidth. To make a comparison, a 
TABLE I. The $\mathrm{VO}_{2}$ band structure properties with different HSE/-U parameters. Previous experimental or calculated references are listed for comparison. Bold values are highlighted as most useful for the interfaces discussed.

\begin{tabular}{lcccc}
\hline \hline $\mathrm{HSE}(\alpha) /-\mathrm{U}(\mathrm{eV})$ parameter & $\mathrm{VO}_{2} \mathrm{E}_{\mathrm{g}}(\mathrm{eV})$ & $\mathrm{V}-d_{\|} \mathrm{O}-p$ gap $(\mathrm{eV})$ & $\mathrm{V}-d_{\| \mid}$bandwidth $(\mathrm{eV})$ & $\mathrm{V}-d_{\|}$peak below E $(\mathrm{eV})$ \\
\hline $0 / 2.5$ & 0.59 & 0.32 & 1.02 & 0.91 \\
$\mathbf{0 . 1 0 / 0}$ & $\mathbf{0 . 5 9}$ & 0.67 & 1.06 & 0.71 \\
$0.25 / 0$ & 2.26 & 0.14 & 0.91 & 0.54 \\
$0.30 / 0$ & 2.89 & 0 & $\ldots$ & $\ldots$ \\
$\mathbf{0 . 3 0 / - 4 . 2}$ & $\mathbf{0 . 6 4}$ & 1.43 & 1.01 & 0.51 \\
Exp. $^{36,37}$ & $0.70^{36}$ & $\sim 1$ & $0.50^{36}$ & $\sim 0.90$ \\
Cal. $^{21,26}$ & $1.1-1.13$ & $0.87-0.95$ & $0.63-0.84$ & $\sim 0.75$ \\
\hline \hline
\end{tabular}

pure GGA+U calculation with an on-site $\mathrm{U}$ of $2.5 \mathrm{eV}$ was also conducted, which produces the correct gap value, matching with previous reports, ${ }^{24}$ but the $\mathrm{V}-d$ O- $p$ separation gap is, however, less close to the experimental data compared to our HSE/-U scheme. Although the band structure details obtained using our HSE/-U scheme are not identical to those obtained from experiments, which is more or less limited by the measurement resolution in the photoemission spectra, ${ }^{36,37}$ the key point here is that our proposed HSE/-U method can reproduce the correct bandgap as well as maintain the basically correct band structure, which is the primary condition for the interface calculation.

To provide the correct bandgap at both sides, the HSE/-U scheme with $0.30 /-4.2 \mathrm{eV}$ parameters is used for the $\mathrm{HfO}_{2}\left(\mathrm{ZrO}_{2}\right)$ and $\mathrm{Al}_{2} \mathrm{O}_{3}$ interfaces, while the $0.25 /-3.0 \mathrm{eV}$ and $0.27 /-4.0 \mathrm{eV}$ pairs are chosen for the $\mathrm{TiO}_{2}$ and $\mathrm{GaN}$ cases. Interface geometries are shown in Fig. 1. It is noted that since our work is based on the supercell calculation, both the oxide side and the $\mathrm{VO}_{2}$ side share the identical calculation parameters as mentioned here. We can see from Fig. 3 that the four proposed interfaces are all insulating, with no gap states appearing. The experimental bandgaps of both $\mathrm{VO}_{2}$ and insulators are well reproduced in the supercell calculations. These dielectric films all form the type-I band alignment with $\mathrm{VO}_{2}$, by straddling its $\mathrm{VBM}$ and conduction band minimum (CBM). The traditional HSE supercell

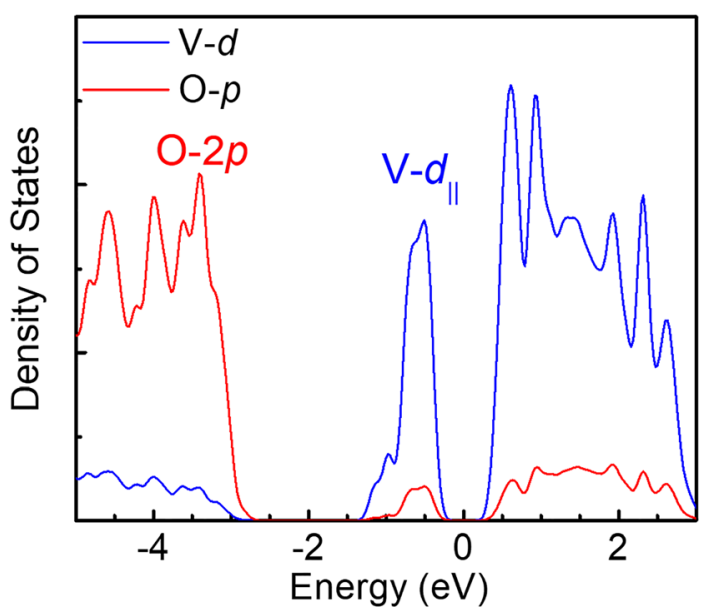

FIG. 2. PDOS of the $\mathrm{V}-3 d$ and $0-2 p$ orbitals of $\mathrm{VO}_{2}$ bulk with the $0.30 /-4.2 \mathrm{eV}$ HSE/-U parameter. The Fermi level is at $0 \mathrm{eV}$. calculation with a $10 \%$ fraction is also calculated (PDOS not shown here), and their band edge energies are shown in Fig. 4. By using the core-level scheme, ${ }^{33}$ the band edge line-up can be determined. Figure 4 summarizes the alignment of $\mathrm{VO}_{2}$ with different dielectrics by two calculation schemes, together with the previous reports. The interface dipole, induced by the charge transfer across the interface, has already been explicitly included in the VBO values obtained by our supercell calculations, as discussed previously.

It is clear that the BOs derived by $\alpha=0.1$ HSE calculations (yellow bars) are not close to the experimental data, owing to the severe underestimation of insulator bandgaps by the small HF fraction. As mentioned earlier, a higher HF fraction will otherwise lead to incorrect properties of $\mathrm{VO}_{2}$, including the bandgap, magnetic ordering, and the latent heat. This makes it less useful.

On the other hand, our proposed HSE/-U method reproduces well the bandgaps on both sides of the interfaces, and based on that, we can have a reliable discussion on the BOs. For the efficient operation of Mott-FET, ${ }^{12}$ the BOs should be larger than $1 \mathrm{eV}$ to confine carriers and suppress leakage currents, similar to that in the MOSFET
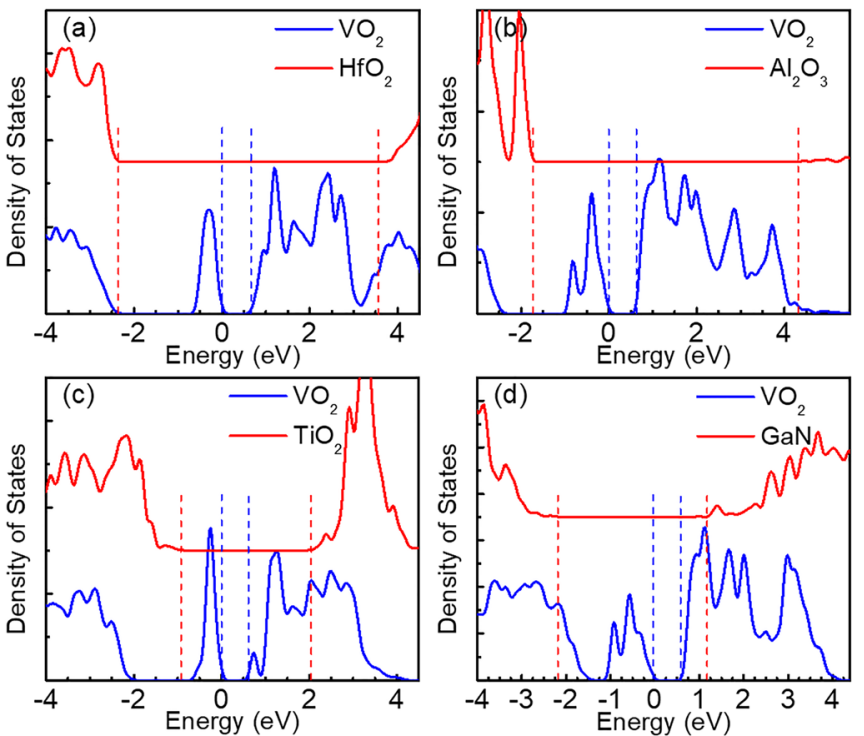

FIG. 3. PDOS of $\mathrm{VO}_{2}$ and dielectric bulk atoms that lie far away from the interfacial region, using the proposed $\mathrm{HSE} /-\mathrm{U}$ scheme. The $\mathrm{VBM}$ of $\mathrm{VO}_{2}$ bulk is referred to $0 \mathrm{eV}$. Band edges are marked by vertical dashed lines. 


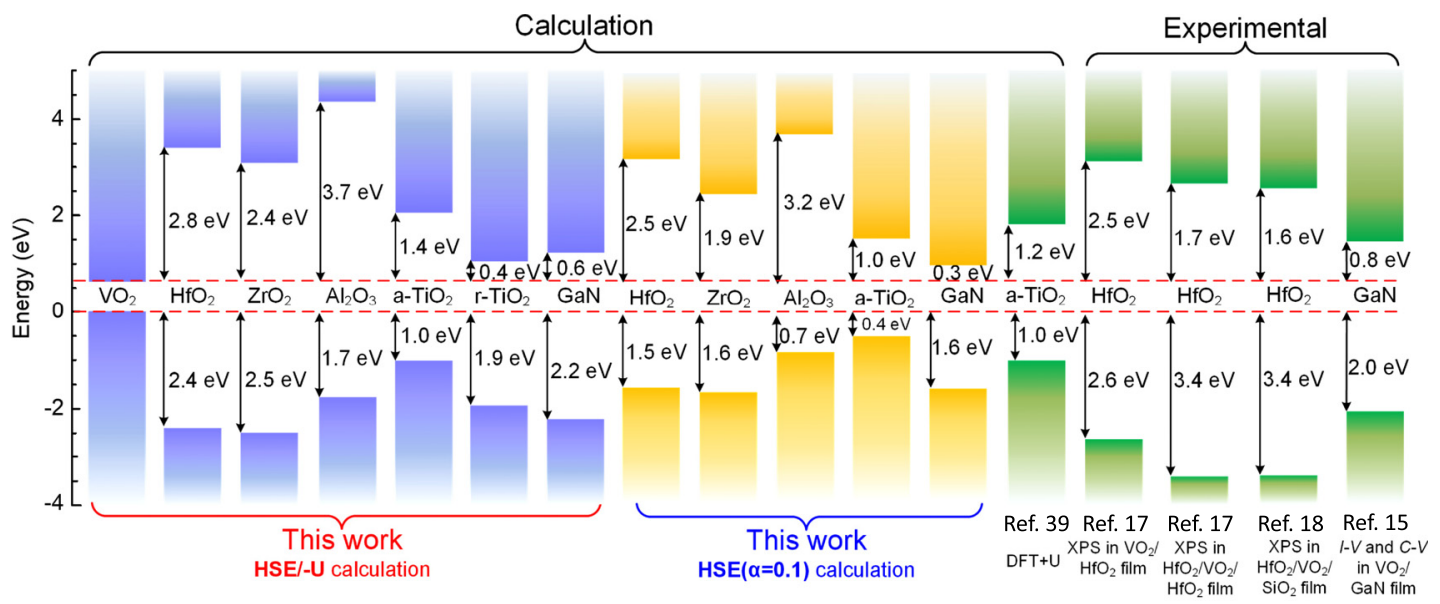

FIG. 4. Schematic band alignment diagram of $\mathrm{VO}_{2}$ with different dielectrics by two calculation methods. a- $\mathrm{TiO}_{2}$ and $\mathrm{r}-\mathrm{TiO}_{2}$ stand for anatase and rutile, respectively. Blue, yellow, and green bars represent the alignment by the proposed hybrid scheme calculation, traditional HSE calculation (this work), and reference/comparison values, respectively.

devices. ${ }^{1,35}$ From the HSE/-U results (blue bars in Fig. 4), we can conclude that in terms of $\mathrm{BO}$, all the studied oxides (except for $\mathrm{r}-\mathrm{TiO}_{2}$ ) can be used as gate insulators for $\mathrm{VO}_{2}$ Mott-FET, for their BOs are all larger than $1 \mathrm{eV}^{35}$ The high- $k$ oxides $\mathrm{HfO}_{2}$ and $\mathrm{ZrO}_{2}$ stand out for they all have the sufficiently large offset $(>2 \mathrm{eV})$, which can effectively confine the channel carriers and suppress gate leakage.

For a- $\mathrm{TiO}_{2}$, the $\mathrm{VBO}$ is somewhat smaller, but the $1 \mathrm{eV}$ offset is already enough for basic device applications. For $\mathrm{r}-\mathrm{TiO}_{2}$, although it also shows a type-I alignment, the $\mathrm{CBO}$ is only $0.4 \mathrm{eV}$, not large enough to confine electrons. Although the band edge of $\mathrm{r}-\mathrm{TiO}_{2}$ is $\sim 1 \mathrm{eV}$ lower than that of $\mathrm{a}-\mathrm{TiO}_{2}$ and in contrast to the experimental report, ${ }^{38}$ it is noted that the edge level is derived from different interface supercells. The considerable band edge variation between $\mathrm{a}-\mathrm{TiO}_{2}$ and $\mathrm{r}-\mathrm{TiO}_{2}$ is due to the extremely large lattice mismatch at the $\mathrm{M} 1-\mathrm{VO}_{2} / \mathrm{r}-\mathrm{TiO}_{2}$ interface. $\mathrm{GaN}$ has a small $\mathrm{CBO}$, thus not suitable for gate insulators, but can be used as the substrate for epitaxial growth of $\mathrm{VO}_{2}{ }^{14}$

The correctness of our proposed HSE/-U calculation scheme is worth verifying. However, most of the studies on dielectric/ $/ \mathrm{VO}_{2}$ interfaces concern the phase transition stability and electronic characteristics, ${ }^{13}$ and only a few focus on the band alignments. ${ }^{15,17,18}$ A brief comparison with the limited reports is summarized in Fig. 4 (green bars). Nemanich et al. found a VBO of $2.6 \mathrm{eV}$ in the $\mathrm{VO}_{2} / \mathrm{HfO}_{2}$ film, ${ }^{17}$ close to our calculated $2.4 \mathrm{eV}$ value. Although they also reported the $3.4 \mathrm{eV} \mathrm{VBO}$ in the $\mathrm{HfO}_{2} / \mathrm{VO}_{2} / \mathrm{HfO}_{2}$ film, this arises from the different configuration and the interfacial dipole has affected the band edge line-up. ${ }^{18}$ Ramanathan et al. fabricated $\mathrm{GaN} / \mathrm{VO}_{2} \mathrm{p}-\mathrm{n}$ heterojunctions with a $\mathrm{CBO}$ of $\sim 0.8 \mathrm{eV}$ by $I-V$ and $C-V$ measurements, ${ }^{15}$ resembling to our calculated $\mathrm{CBO}$ of $0.6 \mathrm{eV}$. Pickett used the $\mathrm{LDA}+\mathrm{U}$ method on the $\mathrm{TiO}_{2} / \mathrm{VO}_{2}$ interface, ${ }^{39}$ and the calculated $\mathrm{VBO}(1.0 \mathrm{eV})$ is the same as our data. No other explicit band alignment values have been reported yet.

Based on our current discussion, the proposed HSE/-U scheme can be well applied for complex interfaces containing narrow gap semiconductors and wide gap insulators. It has distinct advantages over a pure HSE or DFT $+\mathrm{U}$ calculation. To use such a scheme, one first chooses the suitable HF mixing fraction (i.e., $\alpha$ ) to get the correct gap of one bulk material and then tests and determines the correct $U$ value for the other bulk material based on this fraction value. Combining the two parameters, the HSE/-U scheme can be applied for interface supercell calculations. Although this work only focuses on various dielectric $/ \mathrm{VO}_{2}$ interfaces, it should be noted that this scheme can also be employed for other interfaces. For example, for the popular $\mathrm{Si} / \mathrm{ZnO}$ interface that is widely used in solar cells, ${ }^{40}$ the standard HSE hybrid function (with $\alpha=25 \%$ ) will fit the gap of Si, a positive U term on $\mathrm{Zn}-d$ will open up the $\mathrm{ZnO}$ bandgap to $3.4 \mathrm{eV}$, and a correct band alignment with experimental reports is obtained. Besides, a suitable HSE/-U parameter can be considered for high-k oxide coming into contact with the Mott insulator NiO. But for the interface with two wide gap materials, the pure hybrid functional calculation is generally acceptable, as it is widely reported.

In conclusion, we proposed a hybrid calculation scheme that combines a hybrid functional and the reverse GGA+U on complex interface supercell calculations. To verify its reliability, several dielectric films coming into contact with the insulating $\mathrm{VO}_{2}$ are discussed. We find that the experimental bandgaps at both sides are well reproduced and the derived band alignment achieves a good agreement with the experimental reports. Using this $\mathrm{HSE} /-\mathrm{U}$ calculation, we find that oxide insulators including $\mathrm{HfO}_{2}, \mathrm{ZrO}_{2}, \mathrm{Al}_{2} \mathrm{O}_{3}$, and $\mathrm{a}-\mathrm{TiO}_{2}$ all show proper band alignment with the $\mathrm{VO}_{2}$ film, and they are promising for $\mathrm{VO}_{2}$ based Mott-FET applications. This proposed calculation method is suitable for various complex interface supercell calculations.

The authors are grateful to the financial support from the European Union's Horizon 2020 Research and Innovation Program PHASECHANGE SWITCH (Grant No. 737109). We also thank the support from Supercomputing Wales under Project No.SCW1070.

\section{REFERENCES}

${ }^{1} J$. Robertson, J. Vac. Sci. Technol., B 18, 1785 (2000).

${ }^{2}$ Y. Hinuma, A. Grüneis, G. Kresse, and F. Oba, Phys. Rev. B 90, 155405 (2014)

${ }^{3}$ C. Adamo and V. Barone, J. Chem. Phys. 110, 6158 (1999).

${ }^{4}$ A. Alkauskas, P. Broqvist, and A. Pasquarello, Phys. Rev. Lett. 101, 046405 (2008). 
${ }^{5}$ J. Muscat, A. Wander, and N. M. Harrison, Chem. Phys. Lett. 342, 397 (2001). ${ }^{6}$ S. J. Clark and J. Robertson, Phys. Rev. B 82, 085208 (2010).

7J. Heyd, G. E. Scuseria, and M. Ernzerhof, J. Chem. Phys. 124, 219906 (2006).

${ }^{8}$ M. A. L. Marques, J. Vidal, M. J. T. Oliveira, L. Reining, and S. Botti, Phys. Rev. B 83, 035119 (2011).

${ }^{9}$ Y. Hinuma, Y. Kumagai, I. Tanaka, and F. Oba, Phys. Rev. B 95, 075302 (2017).

${ }^{10}$ W. Chen, G. Miceli, G. Rignanese, and A. Pasquarello, Phys. Rev. Mater. 2, 073803 (2018).

${ }^{11}$ K. Liu, S. Lee, S. Yang, O. Delaire, and J. Wu, Mater. Today 21, 875 (2018).

${ }^{12}$ Z. Yang, C. Ko, and S. Ramanathan, Annu. Rev. Mater. Res. 41, 337 (2011).

${ }^{13} \mathrm{~N}$. Shukla, A. V. Thathachary, A. Agrawal, H. Paik, A. Aziz, D. G. Schlom, S. K.

Gupta, R. Engel-Herbert, and S. Datta, Nat. Commun. 6, 7812 (2015).

${ }^{14}$ Y. Zhou and S. Ramanathan, J. Appl. Phys. 112, 074114 (2012).

${ }^{15}$ Y. Zhou and S. Ramanathan, J. Appl. Phys. 113, 213703 (2013).

${ }^{16}$ B. P. Downey, V. D. Wheeler, and D. J. Meyer, Appl. Phys. Express 10, 061101 (2017).

${ }^{17}$ C. Zhu, S. L. Caudle, J. Yang, D. J. Smith, and R. J. Nemanich, J. Vac. Sci. Technol., A 32, 011203 (2014).

${ }^{18}$ C. Zhu, M. Kaur, F. Tang, X. Liu, D. J. Smith, and R. J. Nemanich, J. Appl. Phys. 112, 084105 (2012).

${ }^{19} \mathrm{~S}$. Biermann, A. Poteryaev, A. I. Lichtenstein, and A. Georges, Phys. Rev. Lett. 94, 026404 (2005).

${ }^{20}$ M. Gatti, F. Bruneval, V. Olevano, and L. Reining, Phys. Rev. Lett. 99, 266402 (2007).

${ }^{21} \mathrm{~V}$. Eyert, Phys. Rev. Lett. 107, 016401 (2011).

${ }^{22}$ R. Grau-Crespo, H. Wang, and U. Schwingenschloegl, Phys. Rev. B 86, 081101(R) (2012).

${ }^{23}$ S. Xu, X. Shen, K. A. Hallman, R. F. Haglund, and S. T. Pantelides, Phys. Rev. B 95, 125105 (2017).
${ }^{24} \mathrm{H}$. Lu, Y. Guo, and J. Robertson, Phys. Rev. Mater. 3, 094603 (2019).

${ }^{25} \mathrm{H}$. Lu, Y. Guo, S. J. Clark, and J. Robertson, "Nature of the metal-insulator transition in pure and doped vanadium dioxide" (unpublished).

${ }^{26}$ F. Iori, M. Gatti, and A. Rubio, Phys. Rev. B 85, 115129 (2012).

${ }^{27}$ T. J. Huffman, C. Hendriks, E. J. Walter, J. Yoon, H. Ju, R. Smith, G. L. Carr, H. Krakauer, and M. M. Qazilbash, Phys. Rev. B 95, 075125 (2017).

${ }^{28}$ V. V. Anisimov, J. Zaanen, and O. K. Andersen, Phys. Rev. B 44, 943 (1991).

${ }^{29}$ A. Janotti, D. Segev, and C. G. Van de Walle, Phys. Rev. B 74, 045202 (2006); L. A. Agapito, S. Curtarolo, and M. B. Nardelli, Phys. Rev. X 5, 011006 (2015); N. Tancogne-Dejean, M. J. T. Oliveira, and A. Rubio, Phys. Rev. B 96, 245133 (2017).

${ }^{30}$ S. J. Clark, M. D. Segall, C. J. Pickard, P. J. Hasnip, M. J. Probert, K. Refson, and M. C. Payne, Z. Kristallogr. 220, 567 (2005).

${ }^{31}$ Y. Guo, S. J. Clark, and J. Robertson, J. Chem. Phys. 140, 054702 (2014).

${ }^{32}$ D. Liu, S. J. Clark, and J. Robertson, Appl. Phys. Lett. 96, 032905 (2010).

${ }^{33}$ Z. Zhang, Y. Guo, and J. Robertson, Appl. Phys. Lett. 114, 161601 (2019).

${ }^{34}$ L. Lin and J. Robertson, Appl. Phys. Lett. 98, 082903 (2011).

${ }^{35}$ J. Robertson, J. Vac. Sci. Technol., A 31, 050821 (2013).

${ }^{36}$ S. Shin, S. Suga, M. Taniguchi, M. Fujisawa, H. Kanzaki, A. Fujimori, H. Daimon, Y. Ueda, K. Kosuge, and S. Kachi, Phys. Rev. B 44, 4993 (1990).

${ }^{37}$ K. Okazaki, H. Wadati, A. Fujimori, M. Onoda, Y. Muraoka, and Z. Hiroi, Phys. Rev. B 69, 165104 (2004).

${ }^{38}$ D. O. Scanlon, C. W. Dunnill, J. Buckeridge, S. A. Shevlin, A. J. Logsdail, S. M. Woodley, C. R. A. Catlow, M. J. Powell, R. G. Palgrave, I. P. Parkin, G. W. Watson, T. W. Keal, P. Sherwood, A. Walsh, and A. A. Sokol, Nat. Mater. 12, 798 (2013).

${ }^{39}$ V. Pardo and W. E. Pickett, Phys. Rev. B 81, 035111 (2010).

${ }^{40}$ J. Chen, Z. Zhang, Y. Guo, and J. Robertson, Microelectron. Eng. 216, 111056 (2019). 